\title{
Identification of the key genes and pathways in prostate cancer
}

\author{
SHUTONG FAN ${ }^{1 *}$, ZUMU LIANG $^{1 *}$, ZHIQIN GAO $^{1}$, ZHIWEI PAN $^{2}$, SHAOJIE HAN ${ }^{3}$, XIAOYING LIU $^{1}$, \\ CHUNLING ZHAO $^{1}$, WEIWEI YANG ${ }^{1}$, ZHIFANG PAN ${ }^{1}$ and WEIGUO FENG ${ }^{1}$ \\ ${ }^{1}$ College of Bioscience and Technology, Weifang Medical University, Weifang, Shandong 261053; \\ ${ }^{2}$ Department of Internal Medicine, Laizhou Development Zone Hospital, Yantai, Shandong 261400; \\ ${ }^{3}$ Animal Epidemic Prevention and Epidemic Control Center, Changle County Bureau of Animal Health and Production, \\ Weifang, Shandong 262400, P.R. China
}

Received March 5, 2018; Accepted September 17, 2018

DOI: $10.3892 / \mathrm{ol} .2018 .9491$

\begin{abstract}
Prostate cancer (PCa) is one of the most common malignancies in men globally. The aim of the present study was to identify the key genes and pathways involved in the occurrence of PCa. Gene expression profile (GSE55945) was downloaded from Gene Expression Omnibus, and the differentially expressed genes (DEGs) were identified. Subsequently, Gene ontology analysis, KEGG pathway analysis and protein-protein interaction (PPI) analysis of DEGs were performed. Finally, the identified key genes were confirmed by immunohistochemistry. The GO analysis results showed that the DEGs were mainly participated in cell cycle, cell division, cell development and cell junction. The KEGG pathway analysis showed that the DEGs were mainly enriched in proteoglycans in cancer, endocytosis, focal adhesion and hippo signaling pathway. The PPI analysis results showed that RPS21, FOXO1, BIRC5, POLR2H, RPL22L1 and NPM1 were the key genes involved in the occurrence of $\mathrm{PCa}$, and the Module analysis indicated that the occurrence of PCa was associated with cell cycle, oocyte meiosis and ribosome biogenesis. IHC result showed that the expression of RPS21, BIRC5, POLR2H, RPL22L1 and NPM1 were significantly upregulated in $\mathrm{PCa}$, while the expression of FOXO1 was significantly downregulated in $\mathrm{PCa}$, matching with the bioinformatics analysis. Taken together, several key genes and pathways were identified involved in $\mathrm{PCa}$, which might provide the potential biomarker for prognosis, diagnosis and drug targets.
\end{abstract}

Correspondence to: Dr Weiguo Feng or Dr Zhifang Pan, College of Bioscience and Technology, Weifang Medical University, 7166 Baotong Street, Weifang, Shandong 261053, P.R. China

E-mail: sdaqfwg@126.com

E-mail: sdwfpzf@126.com

${ }^{*}$ Contributed equally

Key words: bioinformatics analysis, prostate cancer, differentially expressed gene, pathways, identification

\section{Introduction}

Prostate cancer (PCa) is one of the most common malignancies in men globally and the second leading cause of cancer associated mortality in developed countries $(1,2)$. Like other cancers, $\mathrm{PCa}$ is considered to be a disease which caused by age, diet and gene aberrations (3). Accumulating evidences have demonstrated that a series of genes and pathways involved in the occurrence, progression and metastasis of PCa (4). At present, the underlying mechanism of $\mathrm{PCa}$ occurrence is still unclear, which limits the diagnosis and therapy. Therefore, it is urgent to identify the key genes and pathways involved in the occurrence of PCa $(2,5)$.

Microarray is a useful tool for analysis of gene expression that can be applied to disease diagnosis and targeted therapy (6,7). During the past decade, hundreds of differentially expressed genes (DEGs) participated in biological processes, cell component, molecular functions and pathways of PCa were identified by microarray technology $(8,9)$. However, previous studies of DEGs analysis have shown relative limitations, for example, no reliable biomarker was identified that could distinguish tumors from normal tissues (10). Therefore, gene expression in the occurrence of PCa needs to be further analyzed by microarray combining bioinformatics technology at present.

In the present study, Gene expression profile (GSE55945) was downloaded from Gene Expression Omnibus (GEO) (http://www.ncbi.nlm.nih.gov/geo/). The gene expression profile was analyzed, and the DEGs were identified between PCa group and normal group. Subsequently, gene ontology (GO) analysis, Kyoto Encyclopedia of Genes and Genomes (KEGG) pathway analysis and protein-protein interaction (PPI) analysis of DEGs were performed. Finally, the expression of screened key genes was verified by immunohistochemistry (IHC). The present study aimed to identify key genes and pathways which involved in the occurrence of $\mathrm{PCa}$ and explored the potential biomarker for prognosis, diagnosis and drug targets.

\section{Materials and methods}

Expression Profile Microarray. Gene expression profile (GSE55945) was downloaded from GEO (http://www.ncbi. nlm.nih.gov/geo/). GSE55945 was based on GPL570 platform (Affymetrix Human Genome U133 Plus 2.0 Array), which 
was submitted by Arredouani et al, and contained 21 samples, including $18 \mathrm{PCa}$ samples and 8 normal prostate samples (8).

Identification of DEGs. The gene expression profile was analyzed by Morpheus online tools (https://software.broadinstitute.org/morpheus/) as previous study (11). The DEGs were identified between PCa group and normal group. The significance of DEGs was identified by classical t-test. The change $\geq$ twofold and $\mathrm{P}<0.05$ was considered to indicate a statistically significant difference.

GO analysis and KEGG pathway analysis of DEGs. In order to analyse the function and pathway of the DEGs, DAVID database (https://david.ncifcrf.gov/) was used for GO analysis and KEGG pathway analysis as previous study (7). $\mathrm{P}<0.05$ was considered to indicate a statistically significant difference.

PPInetworkandmoduleanalysis.Search ToolfortheRetrieval of Interacting Genes (STRING; https://string-db.org/cgi/input.pl) was a database that could assess the protein-protein interaction. The DEGs were mapped to STRING, and a score $>0.4$ was considered to be significant. Then, the Cytoscape software (version 3.3.0) was used to construct PPI networks. Finally, the modules of PPI network were screened by the plug-in Molecular Complex Detection (MCODE). In addition, the pathway analysis was performed in the modules. $\mathrm{P}<0.05$ was considered to indicate a statistically significant difference.

Patients and tissue samples. The tissue microarray was purchased from Alenabio Co., Ltd (Xian, China) including 60 PCa samples from patients and 10 normal prostate tissue samples from healthy donors. The procedures performed in this study involving human patients were in accordance with the ethical standards of the institutional and/or national research committee and with the 1964 Helsinki declaration and its later amendments or comparable ethical standards. The present study was approved by the Research Ethics Committee of Weifang Medical University (Weifang, China).

IHC validation. IHC was performed as previous study (12). The tissue sample was blocked by $0.3 \% \mathrm{H}_{2} \mathrm{O}_{2}$ and blocked by $10 \%$ bovine serum albumin (BSA) for $25 \mathrm{~min}$. Then, the tissue sample was incubated overnight with anti-ribosomal protein S21 (RPS21), anti-forkhead box O1 (FOXO1), anti-baculoviral IAP repeat containing 5 (BIRC5), anti-RNA polymerase II subunit $\mathrm{H}$ (POLR2H), anti-ribosomal protein L22 like 1 (RPL22L1) and anti-nucleophosmin 1 (NPM1; 1:100 dilution; Proteintech, Wuhan, China) at $4^{\circ} \mathrm{C}$ and incubated with secondary antibody (1:1,000 dilution; Beyotime Institute of Biotechnology, Shanghai, China) for $1.5 \mathrm{~h}$ at $30^{\circ} \mathrm{C}$. At last, 3,3-diaminobenzidine (DAB) was used for color visualization and hematoxylin was used for counterstained. In this study, eight pairs of normal and cancerous tissues were stained with each antibody. To evaluate the gene expression, a previously described scoring system was utilized (13). Briefly, the scores of two parameters were multiplied by the staining intensity (range, 0-3) and the percentage of positive cells [range, 0-4 $(0,0-10 \% ; 1,11-25 \% ; 2,26-50 \% ; 3,51-75 \%$; and $4,76-100 \%)]$. The tissue sample with scores of 8 or higher was classified as positive staining. In addition, an independent sample t-test was applied to the results of staining.

\section{Results}

Identification of DEGs. A total of $13 \mathrm{PCa}$ samples and 8 normal samples were analyzed using Morpheus online tools (https://software.broadinstitute.org/morpheus/). A total of 2,000 DEGs were identified in PCa compared to normal group, including 1000 upregulated and 1,000 downregulated genes respectively. The heat map of DEGs expression (including top 40 upregulated and downregulated genes) was shown in Fig. 1.

GO analysis of DEGs. All DEGs were uploaded to DAVID database (https://david.ncifcrf.gov/), and then GO analysis was conducted. The results showed that upregulated DEGs were enriched in biological processes, including cellular response to growth factor stimulus, phosphate metabolic process, cell development, cell cycle and cell division, while downregulated DEGs were enriched in biological processes, including signal transduction, cell communication, response to stimulus, cell projection organization and cell development (Table I). For cell component, upregulated DEGs were enriched in contractile fiber, actin cytoskeleton, anchoring junction, adherens junction and focal adhesion, while downregulated DEGs were enriched in membrane raft, actin cytoskeleton, adherens junction, anchoring junction and membrane-bounded vesicle (Table I). Furthermore, for molecular function, upregulated DEGs were enriched in enzyme binding, cytoskeletal protein binding, enzyme regulator activity, macromolecular complex binding and protein kinase binding, while downregulated DEGs were enriched in RNA binding, cytoskeletal protein binding, enzyme regulator activity, transcription factor binding and calcium ion binding (Table I).

KEGG pathway analysis of DEGs. The KEGG pathway analysis results showed that upregulated DEGs were enriched in proteoglycans in cancer, endocytosis, focal adhesion, hippo signaling pathway and cGMP-PKG signaling pathway, whereas downregulated DEGs were enriched in proteoglycans in cancer, endocytosis, hippo signaling pathway, thyroid hormone signaling pathway and sulfur relay system (Table II).

Module screening from the PPI network. The top 6 hub nodes with high degrees were screened by the STRING database. These hub genes included RPS21, FOXO1, BIRC5, POLR2H, RPL22L1 and NPM1. In addition, total nodes were analyzed by plug-ins MCODE, and the top three significant modules were selected (Fig. 2). The results showed that a total of 91 genes of functional annotation were involved in the modules that associated with cell cycle, oocyte meiosis and ribosome biogenesis in eukaryotes (data not shown).

IHC validation of key genes in PCa samples. To verify the bioinformatics analysis data, the expression of key genes including RPS21, FOXO1, BIRC5, POLR2H, RPL22L1 and NPM1 were examined by IHC in PCa samples and normal samples. As shown in Fig. 3, compared with the normal tissues, the expression of RPS21, BIRC5, POLR2H, RPL22L1 and NPM1 were significantly upregulated in the cancer cells from tumor tissues, while the expression of FOXO1 was significantly 

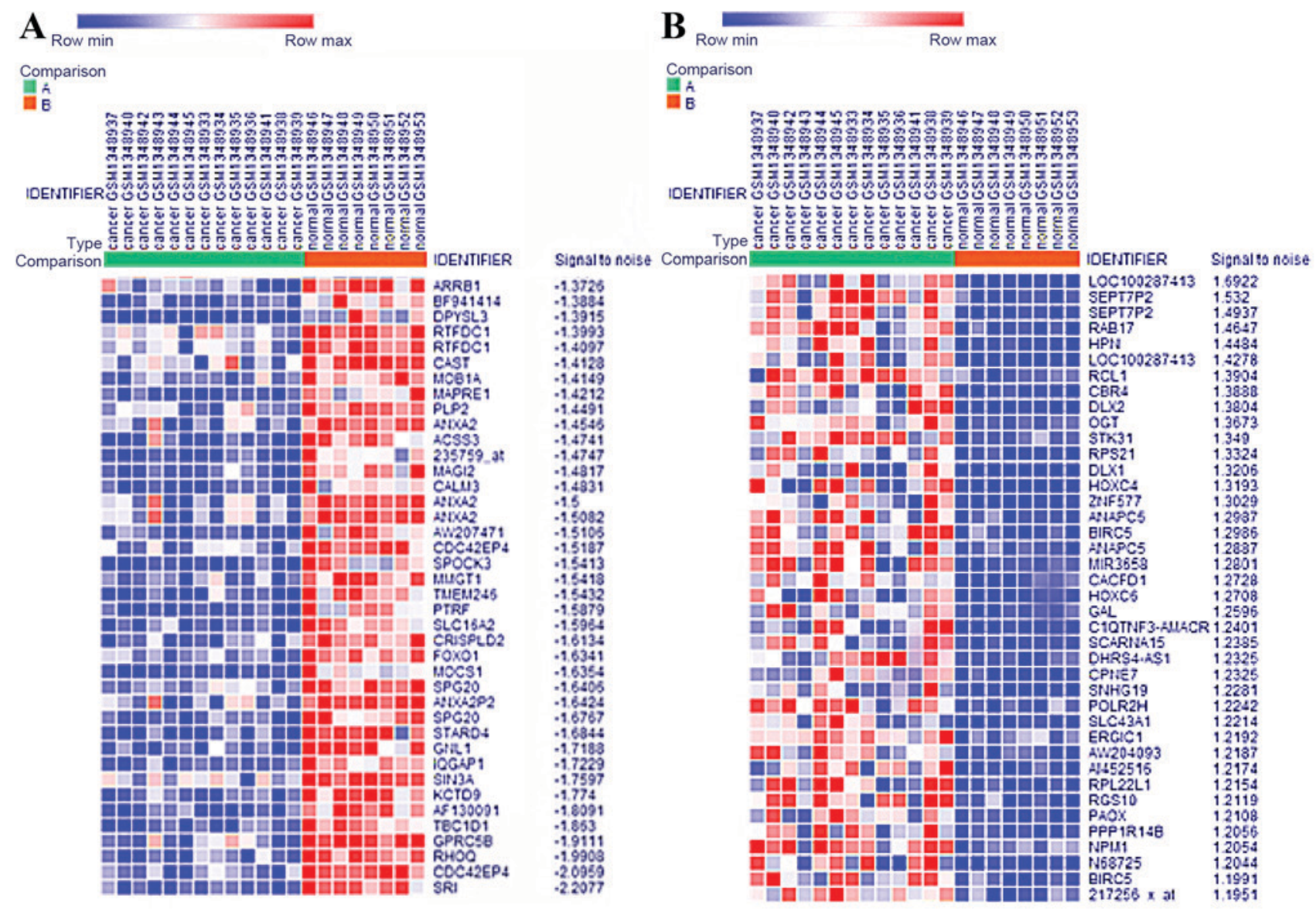

Figure 1. Heatmap of the top 40 upregulated genes and 40 downregulated genes. (A) blue, downregulated; (B) red, upregulated.

downregulated in the cancer cells from tumor tissues $(\mathrm{P}<0.05)$. The IHC results were matched with the bioinformatics analysis.

\section{Discussion}

In the present study, we uploaded GSE21815 and identify 2,000 DEGs (upregulated and downregulated) between PCa and normal tissues by bioinformatics analysis. Go analysis and KEGG pathway analysis showed that the DEGs were mainly involved in cell cycle, cell division, cell development and cell junction. The results of PPI analysis showed that some key genes might play an important role in the occurrence, progression and metastasis of $\mathrm{PCa}$ that could provide the potential biomarker for prognosis, diagnosis and drug targets.

In this study, the gene expression profile of GSE55945 was downloaded from GEO which including 18 PCa samples and 8 normal prostate samples. The results showed that a total of 2,000 DEGs were identified in PCa compared to normal group, including 1,000 upregulated and 1,000 downregulated genes respectively. Previous studies have demonstrated that co-expression genes were frequently involved in similar biological function and signal pathway $(2,9)$. Therefore, GO analysis and KEGG pathway analysis was further performed.

The GO analysis showed that the DEGs were mainly participated in cell cycle, cell division, cell development and cell junction (Table I). This result was consistent with other studies, and the dysregulation of cell function lead to the occurrence, progression and metastasis of PCa $(2,8)$. The KEGG pathway analysis showed that the DEGs were mainly enriched in proteoglycans in cancer, endocytosis, focal adhesion and hippo signaling pathway (Table II). Previous study demonstrated that dysregulation of the hippo pathway exerts a significant impact on cancer development. For example, activated hippo signaling pathway was observed in many types of cancers, including colon, liver, breast, lung and ovary (14). Recent study implied that proteoglycans exert diverse functions in the occurrence of cancer. For instance, proteoglycans contributed to the formation of provisional matrix for tumor growth affecting cell-cell and cell-matrix interactions and signal transduction of tumor cells. Proteoglycans also regulated the phenotype of tumor cells and tumor stroma angiogenesis (15). In addition, other studies showed that endocytosis and focal adhesion were closely related to tumorigenesis $(16,17)$. Bibens-Laulan et al demonstrated that the high expression of galectin-7 in ovarian and breast cancer cells was due to the endocytosis (16). Kanteti et al indicated that focal adhesion kinase play an important role in tumor cell phenotype such as survival, proliferation, migration and invasion (17).

Finally, PPI analysis was performed and the key genes were identified (Fig. 2). Subsequently, the result was confirmed by IHC. As shown in Fig. 3, the expression of RPS21, BIRC5, POLR2H, RPL22L1 and NPM1 were significantly upregulated in $\mathrm{PCa}$, while the expression of FOXO1 was significantly downregulated in PCa. The IHC results were matched with the bioinformatics analysis.

RPS21 was the first identified key gene, belonging to ribosomal proteins (RPS) family. RPS are the pivotal components 
Table I. GO annotation of DEGs in PCa.

A, Upregulated genes

\begin{tabular}{|c|c|c|c|}
\hline Category & Term/gene function & Count & P-value \\
\hline GOTERM_BP_FAT & Cellular response to growth factor stimulus & 27 & $4.7 \times 10^{-6}$ \\
\hline GOTERM_BP_FAT & Regulation of phosphate metabolic process & 25 & $6.2 \times 10^{-6}$ \\
\hline GOTERM_BP_FAT & Cell development & 54 & $6.3 \times 10^{-5}$ \\
\hline GOTERM_BP_FAT & Regulation of cell cycle & 28 & $3.1 \times 10^{-3}$ \\
\hline GOTERM_BP_FAT & Cell division & 19 & $3.7 \times 10^{-3}$ \\
\hline GOTERM_CC_FAT & Contractile fiber & 20 & $5.4 \times 10^{-9}$ \\
\hline GOTERM_CC_FAT & Actin cytoskeleton & 25 & $9.6 \times 10^{-7}$ \\
\hline GOTERM_CC_FAT & Anchoring junction & 31 & $2.5 \times 10^{-6}$ \\
\hline GOTERM_CC_FAT & Adherens junction & 30 & $4.6 \times 10^{-6}$ \\
\hline GOTERM_CC_FAT & Focal adhesion & 21 & $9.4 \times 10^{-6}$ \\
\hline GOTERM_MF_FAT & Enzyme binding & 53 & $6.1 \times 10^{-6}$ \\
\hline GOTERM_MF_FAT & Cytoskeletal protein binding & 31 & $9.0 \times 10^{-3}$ \\
\hline GOTERM_MF_FAT & Enzyme regulator activity & 30 & $9.5 \times 10^{-4}$ \\
\hline GOTERM_MF_FAT & Macromolecular complex binding & 34 & $3.9 \times 10^{-3}$ \\
\hline GOTERM_MF_FAT & Protein kinase binding & 18 & $4.4 \times 10^{-3}$ \\
\hline
\end{tabular}

B, Downregulated genes

\begin{tabular}{|c|c|c|c|}
\hline Category & Term/gene function & Count & P-value \\
\hline GOTERM_BP_FAT & Regulation of signal transduction & 25 & $9.2 \times 10^{-6}$ \\
\hline GOTERM_BP_FAT & Regulation of cell communication & 25 & $3.9 \times 10^{-5}$ \\
\hline GOTERM_BP_FAT & Regulation of response to stimulus & 27 & $4.6 \times 10^{-5}$ \\
\hline GOTERM_BP_FAT & Cell projection organization & 26 & $5.4 \times 10^{-5}$ \\
\hline GOTERM_BP_FAT & Cell development & 33 & $1.0 \times 10^{-4}$ \\
\hline GOTERM_CC_FAT & Membrane raft & 11 & $2.6 \times 10^{-4}$ \\
\hline GOTERM_CC_FAT & Actin cytoskeleton & 12 & $2.6 \times 10^{-3}$ \\
\hline GOTERM_CC_FAT & Adherens junction & 14 & $7.5 \times 10^{-3}$ \\
\hline GOTERM_CC_FAT & Anchoring junction & 14 & $9.1 \times 10^{-3}$ \\
\hline GOTERM_CC_FAT & Membrane-bounded vesicle & 44 & $1.2 \times 10^{-3}$ \\
\hline GOTERM_MF_FAT & RNA binding & 43 & $9.3 \times 10^{-6}$ \\
\hline GOTERM_MF_FAT & Cytoskeletal protein binding & 17 & $1.1 \times 10^{-4}$ \\
\hline GOTERM_MF_FAT & Enzyme regulator activity & 18 & $2.3 \times 10^{-3}$ \\
\hline GOTERM_MF_FAT & Transcription factor binding & 11 & $7.7 \times 10^{-3}$ \\
\hline GOTERM_MF_FAT & Calcium ion binding & 12 & $2.9 \times 10^{-3}$ \\
\hline
\end{tabular}

GO, gene ontology; PCa, prostate cancer; DEGs, differentially expressed genes.

of ribosome, which associated with proliferation, differentiation, DNA repair and apoptosis of cell (18). Arthurs et al reported that RPS21 was upregulated in PCa and might serve to be a possible biomarker (19), which was correspond to our study. To our knowledge, this was the only study of RPS 21 in cancer up to now. Moreover, Huang et al reported that RPS27L may be a useful index for predicting prognoses in colorectal cancer (20). Li et al also reported that knockdown of RPSL26 or RPSL29 significantly inhibits cell proliferation in pancreatic cancer (21). The second key gene was FOXO1, belonging to the FOXOs family, which involved in cell proliferation, differentiation and apoptosis by the regulation of multiple genes (22). Consistent with our study, previous studies demonstrated the expression of FOXO1 was downregulated in PCa $(23,24)$. Moreover, other studies further proposed that FOXO1 was a key tumor suppressor in cancer, including PCa $(22,25)$. The third identified key gene was BIRC5, which play an important role in the occurrence and progression of cancer (26). Wang et al reported that BIRC5 was involved in the tumorigenesis of colorectal cancer (27). In addition, BIRC5 was reported to be associated with microtubule-kinetochore attachment, interacting with cell adhesion $(28,29)$. Therefore, BIRC5 might play a critical role in metastasis of PCa. The fourth identified key gene was POLR2H, which was the necessary subunit of RNA polymerase II, which 
Table II. KEGG pathway analysis of DEGs in PCa.

A, Upregulated genes

\begin{tabular}{|c|c|c|c|}
\hline KEGG terms & Count & P-value & Genes \\
\hline Proteoglycans in cancer & 13 & $5.0 \times 10^{-5}$ & $\begin{array}{l}\text { IQGAP1, ROCK2, ARHGEF12, TIMP3, CAV1, CAV2, FZD1, } \\
\text { HOXD10, PAK1, PPP1R12A, PPP1R12B, SDC4, TGFB2 }\end{array}$ \\
\hline Endocytosis & 12 & $1.9 \times 10^{-4}$ & $\begin{array}{l}\text { SH3GLB1, VPS37A, ARRB1, CAV1, CAV2, CHMP1B, CHMP7, } \\
\text { SNX2, SPG20, TGFB2, TGFB3, VPS36 }\end{array}$ \\
\hline Focal adhesion & 10 & $4.2 \times 10^{-3}$ & $\begin{array}{l}\text { ROCK2, CAV1, CAV2, COL4A6, CCND2, MYLK, PAK1, PPP1R12A, } \\
\text { PPP1R12B, VCL }\end{array}$ \\
\hline Hippo signaling pathway & 8 & $8.3 \times 10^{-3}$ & MOB1A, WWTR1, YAP1, CCND2, FZD1, SNAI2, TGFB2, TGFB3 \\
\hline cGMP-PKG signaling pathway & 8 & $1.4 \times 10^{-3}$ & $\begin{array}{l}\text { GNAI2, ROCK2, CALM1, CALM3, MEF2A, MYLK, NPR2, } \\
\text { PPP1R12A }\end{array}$ \\
\hline
\end{tabular}

B, Downregulated genes

\begin{tabular}{lccl}
\hline KEGG terms & Count & P-value & Genes \\
\hline Proteoglycans in cancer & 8 & $1.6 \times 10^{-3}$ & IQGAP1, ARHGEF12, TIMP3, CAV1, CAV2, FZD1, HOXD10, SDC4 \\
Endocytosis & 6 & $7.2 \times 10^{-3}$ & SH3GLB1, VPS37A, ARRB1, CAV1, CAV2, SPG20 \\
Hippo signaling pathway & 5 & $4.2 \times 10^{-2}$ & MOB1A, WWTR1, YAP1, FZD1, SNAI2 \\
Thyroid hormone signaling pathway & 4 & $7.6 \times 10^{-2}$ & SIN3A, FOXO1, RXRA, SLC16A2 \\
Sulfur relay system & 2 & $8.4 \times 10^{-2}$ & MOCS1, MOCS2
\end{tabular}

KEGG, Kyoto Encyclopedia of Genes and Genomes; PCa, prostate cancer; DEGs, differentially expressed genes; FOXO1, forkhead box O1.

A

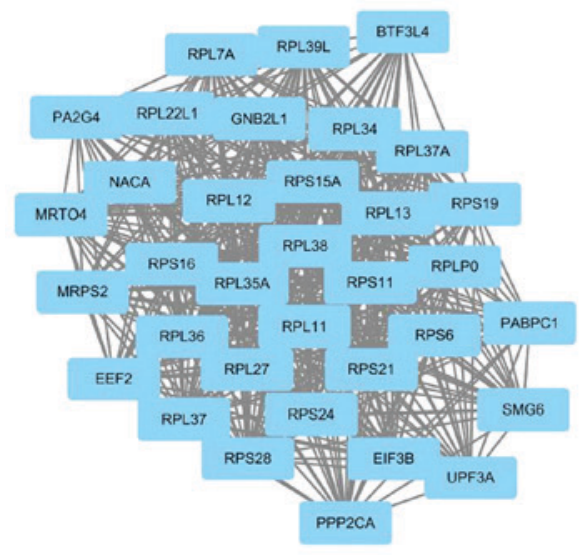

B

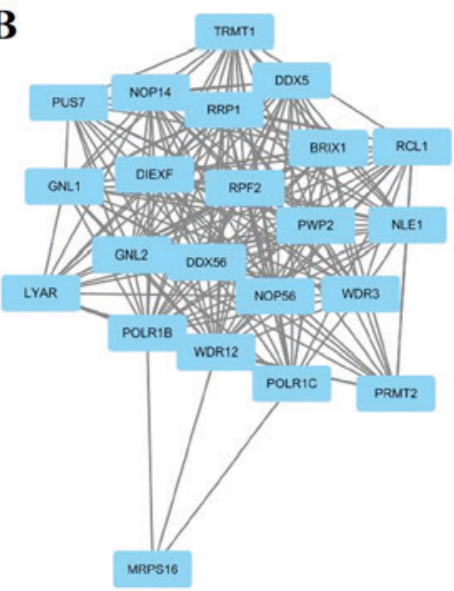

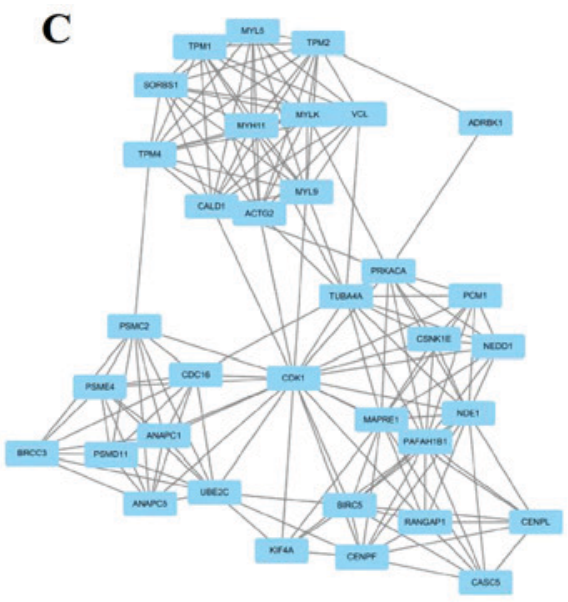

Figure 2. Top three modules from the PPI network. (A) module1, (B) module2, (C) module3. PPI, protein-protein interaction.

was essential for transcription of DNA (30). To our knowledge, the related study of POLR2H was extremely rare. This study indicated that POLR2H was involved in the occurrence and progression of $\mathrm{PCa}$ for the first time, and the mechanism need to be further explored. The fifth key gene was RPL22L1, which was identified as a trace component of ribosome $(31,32)$. Wu et al reported that RPL22L1 could promote ovarian cancer metastasis by inducing epithelial-to-mesenchymal transition (33). Moreover, recent study also demonstrated that RPL22L1 could play vital and definite roles in hematopoietic development (34). The sixth key gene was NPM1, which play an crucial role in cell growth and proliferation (35). Leotoing ea al reported that NPM1 was significantly upregulated in prostate tumour cells, indicating that NPM1 might be an enhancer in progression of PCa $(36,37)$. Further study showed that NPM1 was critical for migration and invasion of $\mathrm{PCa}$, and knockdown of NPM1 resulted in a decrease in the growth of the tumor cell (35).

Module analysis of PPI indicated that the occurrence of PCa was associated with cell cycle, oocyte meiosis and ribosome biogenesis. It is well known that cell cycle is involved in the occurrence, progression and metastasis of cancer (38-40). Moreover, previous studies demonstrated that ribosome biogenesis was closely related to tumorigenesis, and suppressing ribosome biogenesis could inhibit cancer development $(41,42)$. 
A1

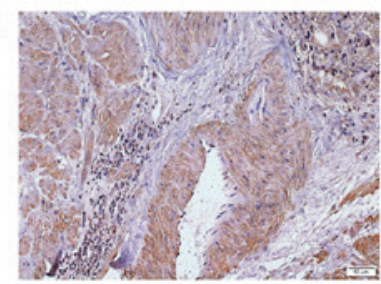

A2

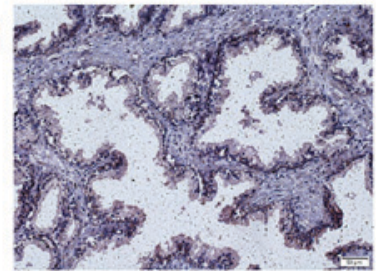

D1

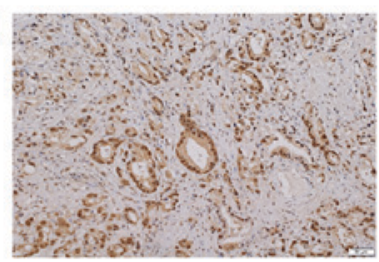

D2

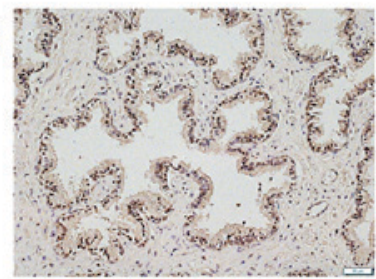

B1

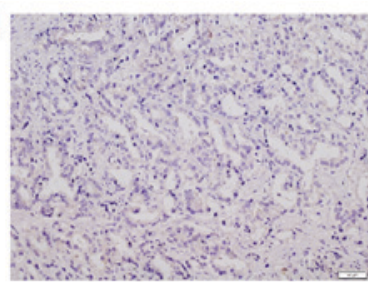

B2

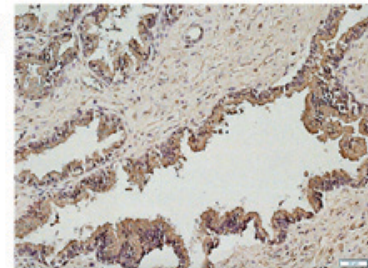

E1

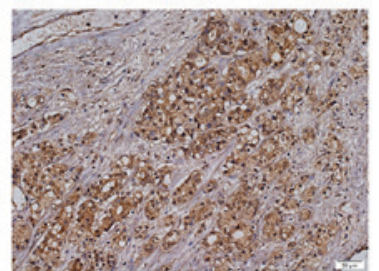

E2

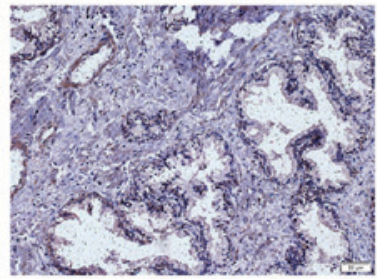

C1

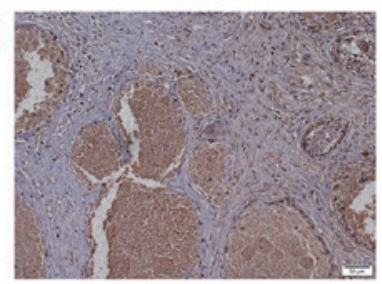

C2

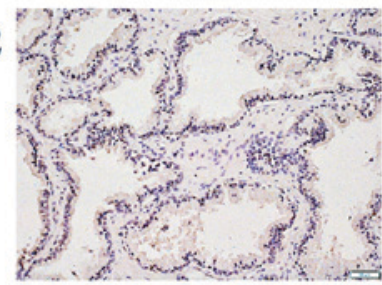

F1

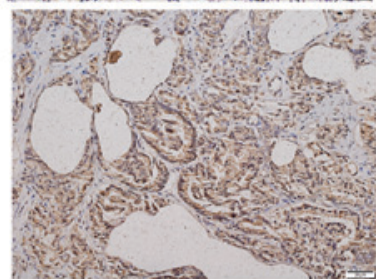

F2

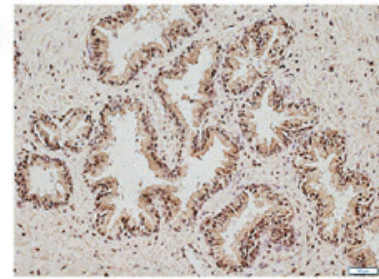

Figure 3. The expression of DEGs in PCa compared to normal tissues. (A1) RPS21 of PCa; (A2) RPS21 of control; (B1) FOXO1 of PCa; (B2) FOXO1 of control; (C1) BIRC5 of PCa; (C2) BIRC5 of control; (D1) POLR2H of PCa; (D2) POLR2H of control; (E1) RPL22L1 of PCa; (E2) RPL22L1 of control; (F1) NPM1 of PCa; (F2) NPM1 of control. IHC, magnification, x400. Scale bar, $50 \mu \mathrm{m}$. Control: Normal tissues. DEGs, differentially expressed genes; PCa, prostate cancer; IHC, immunohistochemistry; FOXO1, forkhead box O1; BIRC5, baculoviral IAP repeat containing 5; RPS21, ribosomal protein S21; POLR2H, RNA polymerase II subunit H; RPL22L1, ribosomal protein L22 like 1; NPM1, nucleophosmin 1.

Therefore, drugs targeted ribosome biogenesis was proposed recently for cancer therapy, which could repress tumor cell proliferation without genotoxic activity (43).

In conclusion, our study identified some key genes and pathways by bioinformatics analysis, which might be involved in the occurrence of PCa. The molecular mechanism of these key genes in the occurrence of PCa need to be further studied.

\section{Acknowledgements}

Not applicable.

\section{Funding}

The present study was supported by Natural Science Foundation of Shandong Province (grant nos. ZR2013CM032, ZR2014CL034, ZR2015HM028 and ZR2017MH103), Science and Technology Development Plan of Shandong Province (grant no. 2015GSF118178) and Medical and Health Development Plan of Shandong Province (grant no. 2017WS058).

\section{Availability of data and materials}

The datasets generated and/or analyzed during the current study are available in the GSE55945 repository, https://www. ncbi.nlm.nih.gov/geo/.

\section{Authors' contributions}

WF and ZFP designed the study. SF and ZL performed the data analysis and wrote the paper. ZG and ZWP identified the DEGs. SH and XL carried out the GO analysis, KEGG pathway analysis and PPI analysis. CZ and WY carried out immunohistochemical analysis. All the authors have read and approved the final manuscript.

\section{Ethics approval and consent to participate}

The procedures performed in this study involving human patients were in accordance with the ethics standards of the institutional and/or national research committee and with the 1964 Helsinki declaration and its later amendments or comparable ethical standards. The present study was approved by the Research Ethics Committee of Weifang Medical University. Written informed consent was obtained from all patients.

\section{Patient consent for publication}

Not applicable.

\section{Competing interests}

The authors declare that they have no competing interests. 


\section{References}

1. Djulbegovic M, Beyth RJ, Neuberger MM, Stoffs TL, Vieweg J, Djulbegovic B and Dahm P: Screening for prostate cancer: Systematic review and meta-analysis of randomised controlled trials. BMJ 341: c4543, 2010

2. Liu L, Guo K, Liang Z, Li F and Wang H: Identification of candidate genes that may contribute to the metastasis of prostate cancer by bioinformatics analysis. Oncol Lett 15: 1220-1228, 2018.

3. Abate-Shen C and Shen MM: Molecular genetics of prostate cancer. Genes Dev 14: 2410-2434, 2000.

4. Cancer genome atlas research network: The molecular taxonomy of primary prostate cancer. Cell 163: 1011-1025, 2015.

5. Hafeez BB, Zhong W, Fischer JW, Mustafa A, Shi X, Meske L, Hong H, Cai W, Havighurst T, Kim K and Verma AK: Plumbagin, a medicinal plant (Plumbago zeylanica)-derived 1,4-naphthoquinone, inhibits growth and metastasis of human prostate cancer PC-3M-luciferase cells in an orthotopic xenograft mouse model. Mol Oncol 7: 428-439, 2013.

6. Nannini M, Pantaleo MA, Maleddu A, Astolfi A, Formica S and Biasco G: Gene expression profiling in colorectal cancer using microarray technologies: Results and perspectives. Cancer Treat Rev 35: 201-209, 2009.

7. Liang $\mathrm{B}$, Li C and Zhao J: Identification of key pathways and genes in colorectal cancer using bioinformatics analysis. Med Oncol 33: 111, 2016.

8. Arredouani MS, Lu B, Bhasin M, Eljanne M, Yue W, Mosquera JM, Bubley GJ, Li V, Rubin MA, Libermann TA and Sanda MG: Identification of the transcription factor single-minded homologue 2 as a potential biomarker and immunotherapy target in prostate cancer. Clin Cancer Res 15: 5794-5802, 2009.

9. Marín-Aguilera M, Codony-Servat J, Kalko SG, Fernández PL, Bermudo R, Buxo E, Ribal MJ, Gascón P and Mellado B: Identification of docetaxel resistance genes in castration-resistant prostate cancer. Mol Cancer Ther 11: 329-339, 2012.

10. Lascorz J, Hemminki K and Försti A: Systematic enrichment analysis of gene expression profiling studies identifies consensus pathways implicated in colorectal cancer development. J Carcinog 10: 7, 2011.

11. Mi B, Liu G, Zhou W, Lv H, Liu Y and Liu J: Identification of genes and pathways in the synovia of women with osteoarthritis by bioinformatics analysis. Mol Med Rep 17: 4467-4473, 2018.

12. Feng W, Zhou D, Meng W, Li G, Zhuang P, Pan Z, Wang G and Cheng Z: Growth retardation induced by avian leukosis virus subgroup $J$ associated with down-regulated Wnt/ $\beta$-catenin pathway. Microb Pathog 104: 48-55, 2017.

13. Wang W, Zhang J, Zhan X, Lin T, Yang M, Hu J, Han B and Hu S: SOX4 is associated with poor prognosis in cholangiocarcinoma. Biochem Biophys Res Commun 452: 614-621, 2014.

14. Yu FX, Zhao B and Guan KL: Hippo pathway in organ size control, tissue homeostasis, and cancer. Cell 163: 811-828, 2015.

15. Theocharis AD and Karamanos NK: Proteoglycans remodeling in cancer: Underlying molecular mechanisms. Matrix Biol pii: S0945-053X: 30313-X, 2017.

16. Bibens-Laulan N and St-Pierre Y: Intracellular galectin-7 expression in cancer cells results from an autocrine transcriptional mechanism and endocytosis of extracellular galectin-7. PLoS One 12: e0187194, 2017.

17. Kanteti R, Mirzapoiazova T, Riehm JJ, Dhanasingh I, Mambetsariev B, Wang J, Kulkarni P, Kaushik G, Seshacharyulu P, Ponnusamy MP, et al: Focal adhesion kinase a potential therapeutic target for pancreatic cancer and malignant pleural mesothelioma. Cancer Biol Ther 19: 316-327, 2018.

18. Wang W, Nag S, Zhang X, Wang MH, Wang H, Zhou J and Zhang R: Ribosomal proteins and human diseases: Pathogenesis, molecular mechanisms, and therapeutic implications. Med Res Rev 35: 225-285, 2015.

19. Arthurs C, Murtaza BN, Thomson C, Dickens K, Henrique R, Patel HRH, Beltran M, Millar M, Thrasivoulou C and Ahmed A: Expression of ribosomal proteins in normal and cancerous human prostate tissue. PLoS One 12: e0186047, 2017.

20. Huang CJ, Yang SH, Lee CL, Cheng YC, Tai SY and Chien CC: Ribosomal protein S27-like in colorectal cancer: A candidate for predicting prognoses. PLoS One 8: e67043, 2013.

21. Li C, Ge M, Yin Y, Luo M and Chen D: Silencing expression of ribosomal protein L26 and L29 by RNA interfering inhibits proliferation of human pancreatic cancer PANC-1 cells. Mol Cell Biochem 370: 127-139, 2012.
22. Duan X, Kong Z, Liu Y, Zeng Z, Li S, Wu W, Ji W, Yang B, Zhao Z and Zeng $\mathrm{G}$ : $\beta$-arrestin 2 contributes to cell viability and proliferation via the down-regulation of FOXO1 in castration-resistant prostate cancer. J Cell Physiol 230: 2371-2381, 2015.

23. Dong XY, Chen C, Sun X, Guo P, Vessella RL, Wang RX, Chung LW, Zhou W and Dong JT: FOXO1A is a candidate for the 13 q14 tumor suppressor gene inhibiting androgen receptor signaling in prostate cancer. Cancer Res 66: 6998-7006, 2006.

24. Yu JJ, Wu YX, Zhao FJ and Xia SJ: miR-96 promotes cell proliferation and clonogenicity by down-regulating of FOXO1 in prostate cancer cells. Med Oncol 31: 910, 2014.

25. Fu Z and Tindall DJ: FOXOs, cancer and regulation of apoptosis. Oncogene 27: 2312-2319, 2008.

26. Altieri DC: Survivin, cancer networks and pathway-directed drug discovery. Nat Rev Cancer 8: 61-70, 2008.

27. Wang H, Zhang X, Wang L, Zheng G, Du L, Yang Y, Dong Z, Liu Y, Qu A and Wang C: Investigation of cell free BIRC5 mRNA as a serum diagnostic and prognostic biomarker for colorectal cancer. J Surg Oncol 109: 574-579, 2014.

28. Vitale I, Galluzzi L, Senovilla L, Criollo A, Jemaà M, Castedo M and Kroemer G: Illicit survival of cancer cells during polyploidization and depolyploidization. Cell Death Differ 18: 1403-1413, 2011.

29. Kwon M, GodinhoSA, Chandhok NS, Ganem NJ, Azioune A, Thery M and Pellman D: Mechanisms to suppress multipolar divisions in cancer cells with extra centrosomes. Genes Dev 22: 2189-2203, 2008.

30. Du YJ, Hou YL and Hou WR: Molecular characterization of a gene POLR2H encoded an essential subunit for RNA polymerase II from the Giant Panda (Ailuropoda Melanoleuca). Mol Biol Rep 40: 1495-1498, 2013.

31. Sugihara Y, Honda H, Iida T, Morinaga T, Hino S, Okajima T, Matsuda T and Nadano D: Proteomic analysis of rodent ribosomes revealed heterogeneity including ribosomal proteins L10-like, L22-like 1, and L39-like. J Proteome Res 9: 1351-1366, 2010.

32. O'Leary MN, Schreiber KH, Zhang Y, Duc AC, Rao S, Hale JS, Academia EC, Shah SR, Morton JF, Holstein CA, et al: The ribosomal protein Rpl22 controls ribosome composition by directly repressing expression of its own paralog, Rpl2211. PLoS Genet 9: e1003708, 2013.

33. Wu N, Wei J, Wang Y, Yan J, Qin Y, Tong D, Pang B, Sun D, Sun H, Yu Y, et al: Ribosomal L22-like1 (RPL22L1) promotes ovarian cancer metastasis by inducing epithelial-to-mesenchymal transition. PLoS One 10: e0143659, 2015.

34. Zhang Y, Duc AC, Rao S, Sun XL, Bilbee AN, Rhodes M, Li Q, Kappes DJ, Rhodes J and Wiest DL: Control of hematopoietic stem cell emergence by antagonistic functions of ribosomal protein paralogs. Dev Cell 24: 411-425, 2013.

35. Loubeau G, Boudra R, Maquaire S, Lours-Calet C, Beaudoin C, Verrelle $\mathrm{P}$ and Morel L: NPM1 silencing reduces tumour growth and MAPK signalling in prostate cancer cells. PLoS One 9: e96293, 2014.

36. Léotoing L, Meunier L, Manin M, Mauduit C, Decaussin M, Verrijdt G, Claessens F, Benahmed M, Veyssière G, Morel L and Beaudoin C: Influence of nucleophosmin/B23 on DNA binding and transcriptional activity of the androgen receptor in prostate cancer cell. Oncogene 27: 2858-2867, 2008.

37. Dai L, Li J, Xing M, Sanchez TW, Casiano CA and Zhang JY: Using serological proteome analysis to identify serum anti-nucleophosmin 1 autoantibody as a potential biomarker in european-american and african-american patients with prostate cancer. Prostate 76: 1375-1386, 2016.

38. Yun SJ, Moon SK and Kim WJ: Investigational cell cycle inhibitors in clinical trials for bladder cancer. Expert Opin Investig Drugs 22: 369-377, 2013.

39. Zhao Z, Liu J, Wang C, Wang Y, Jiang Y and Guo M: MicroRNA-25 regulates small cell lung cancer cell development and cell cycle through cyclin E2. Int J Clin Exp Pathol 7: 7726-7734, 2014.

40. Soták M, Sumová A and Pácha J: Cross-talk between the circadian clock and the cell cycle in cancer. Ann Med 46: 221-232, 2014.

41. Gentilella A, Kozma SC and Thomas G: A liaison between mTOR signaling, ribosome biogenesis and cancer. Biochim Biophys Acta 1849: 812-820, 2015.

42. Zhang C, Yin C, Wang L, Zhang S, Qian Y, Ma J, Zhang Z, Xu Y and Liu S: HSPC111 governs breast cancer growth by regulating ribosomal biogenesis. Mol Cancer Res 12: 583-594, 2014.

43. Brighenti E, Treré D and Derenzini M: Targeted cancer therapy with ribosome biogenesis inhibitors: A real possibility? Oncotarget 6: 38617-38627, 2015.

This work is licensed under a Creative Commons Attribution-NonCommercial-NoDerivatives 4.0 International (CC BY-NC-ND 4.0) License. 